\title{
ANXA5 Enhances Malignancy of Murine Hepatocarcinoma Hca-P Cells via ERK Activation and E-cadherin Suppression
}

\author{
Shuqing Liu ${ }^{\dagger}$, Chen Chen ${ }^{\dagger}$, Mingzhong Sun* \\ College of Basic Medicinal Sciences, Dalian Medical University, Dalian, China \\ Email address: \\ lsqsmz@163.com (Shuqing Liu),644734338@qq.com (Chen Chen),smzlsq@163.com (Mingzhong Sun) \\ ${ }^{*}$ Corresponding author \\ ${ }^{\dagger}$ Shuqing Liu and Chen Chen are co-first authors.
}

To cite this article:

Shuqing Liu, Chen Chen, Mingzhong Sun. ANXA5 Enhances Malignancy of Murine Hepatocarcinoma Hca-P Cells via ERK Activation and E-cadherin Suppression. American Journal of Life Sciences. Vol. 7, No. 1, 2019, pp. 31-37. doi: 10.11648/j.ajls.20190701.16

Received: April 1, 2019; Accepted: May 20, 2019; Published: May 23, 2019

\begin{abstract}
The invasion and metastasis are linked to the rapid progression and poor prognosis of hepatocarcinoma patients. Lymphatic metastasis is potentially involved in above pathogenesis with unclear mechanism. Previously, we found the deregulation of annexin A5 (ANXA5), a member of $\mathrm{Ca}^{2+}$-regulated phospholipid- and membrane-binding annexin family protein, mediated the in vivo malignancy, lymph node metastasis (LNM) rate and level of mice transplanted with Hca-P, a murine hepatocarcinoma cell line with the LNM potential rate of $\sim 25 \%$. Current work aimed to investigate the influence with action mechanism of ANXA5 overexpression on the in vitro malignant behaviours of Hca-P cells. For overexpressing ANXA5, the Anxa5 gene was ligated into pCDNA3.1 (+) vector and transfected into Hca-P named as Hca-P-ANXA5up. Hca-P transfected with empty pCDNA3.1 $(+)$ vector was named as Hca-P-mock and used as the control. The monoclonal Hca-P-mock and Hca-P-ANXA5up cell lines were obtained against G418 screening using limiting dilution method. Compared with the Hca-P-mock cells, Western blotting assay indicated ANXA5 expression level was increased by $50.1 \%(p=0.025)$ in the Hca-P-ANXA5up cells. Transwell chamber assays indicated that the migration and invasion capacities of Hca-P-ANXA5up cells were increased by $150.2 \%(p=0.001)$ and $94.8 \%(p=0.003)$ than Hca-P-mock cells. ANXA5 overexpression enhanced the levels of p-MEK (Ser217/221), ERK1, ERK2, p-ERK1 (Thr202/Tyr204) and p-ERK2 (Thr185/Tyr187), and suppressed the levels of E-Cadherin, Snail and Slug in Hca-P cells. Current work shows ANXA5 overexpression enhances the malignant behaviours of hepatocarcinoma Hca-P cells through activating p-MEK-ERK pathway and suppressing E-Cadherin, Snail and Slug. It is of potential value in tumor malignancy and lymphatic metastasis of hepatocarcinoma.
\end{abstract}

Keywords: Hepatocarcinoma, ANXA5, ERK, E-Cadherin

\section{Introduction}

Hepatocarcinoma seriously affects human health and life quality due to its rapid progression and poor prognosis. It ranks the third among the causes of cancer-related deaths [1]. At present, surgical excision is still the main treatment for liver cancer. However, high postoperative recurrence was the leading death cause of patients [2]. Metastasis leads to poor prognosis of hepatocarcinoma [3, 4]. Intrahepatic metastasis accounts for $70 \%$ of recurrent liver cancer [3]. Lymphatic metastasis results in a $50 \%$ reduced prognosis of cancer patients $[5,6]$. The molecular mechanism of tumor lymphatic metastasis remains unclear. The study on lymphatic metastasis contributes to better management for hepatocarcinoma.

Anxa5 is a member of $\mathrm{Ca}^{2+}$-regulated phospholipid-binding annexin superfamily [7-9]. Its dysexpression is linked to the development, metastasis and drug-resistance of various cancers. Previous work from our lab using a pair of syngenetic murine hepatocarcinoma ascites cell lines, Hca-F with $75 \%$ and Hca-P with 25\% LNM rates [10-14], indicated ANXA5 was a potential promoter in murine hepatocarcinoma tumorigenicity and lymphatic metastasis. ANXA5 upregulation enhanced the tumorigenicity, LNM rate and level of Hca-P-transplanted mice [7, 14]. ERK pathway participates in the proliferation, differentiation, migration and invasion of tumor cells [15]. Elevated ERK signal transduction promoted hepatocarcinoma [16]. ANXA5 suppressed ERK via 
deactivating Shc and Grb2 in breast cancer MCF-7 cells [17]. Current work raveled ANXA5 overexpression enhanced the in vitro migration and invasion of Hca-P cells via activating p-MEK-ERK and suppressing E-Cadherin, Snail and Slug.

\section{Material and Methods}

\section{Cell culture}

Murine hepatocarcinoma Hca-P cell line was created and maintained by our group. Hca-P cells were incubated in RPMI-1640 (Gibco, US) supplemented with 15\% FBS (TransGen, China) in a humidified environment with $5 \% \mathrm{CO}_{2}$ at $37^{\circ} \mathrm{C}$.

Monoclonal Hca-P cell line with ANXA5 overexpression

Anxa5 PCR amplification primers, F: 5'-CGGGATCCATC ATGGCTACGAGAGGCACTGTGAC-3' and R: 5'-GGAAT TCTGTCATCCTCGCCCCCGCAGAGC-3', were designed by Oligo7. Total RNA was extracted from Hca-P cells using Trizol $^{\mathrm{TM}}$ reagent (Life Technologies, US) and amplified by RT-PCR using PrimeScript RT-PCR Kit (Takara, Japan). PCR was performed on a MyCycle ${ }^{\mathrm{TM}}$ Thermal Cycler (Bio-Rad, US). Anxa5 PCR product was purified using universal DNA purification kit (Tiangen, China), inserted with an A base tail using Ex Taq ${ }^{\circledR}$ HS kit and ligated into a pMD ${ }^{\circledR} 19-\mathrm{T}$ simple vector (TaKaRa, Japan). The recombinant $\mathrm{pMD}^{\circledR} 19-\mathrm{T}-$ Anxa5 plasmid was amplified in LB medium with $100 \mu \mathrm{g} / \mathrm{mL}$ ampicillin (Sigma, US). The vector was subcloned into a pcDNA3.1 $(+)$ vector, transformed into competent $E$. coli cells and amplified for plasmid extraction by E.Z.N.A. ${ }^{\circledR}$ Endo-Free Plasmid Mini Kit (OMEGA, US). Recombinant plasmids were finally validated by sequencing analysis.

Hca-P cells in $15 \%$ FBS were seeded into a $24-w e l l$ plate at $10^{5}$ cells $/ 500 \mu \mathrm{L}$ per well at $37^{\circ} \mathrm{C}$ with $5 \% \mathrm{CO}_{2}$ for $24 \mathrm{~h}$. Then, each of the recombinant and empty plasmids were mixed with Lipofectamine $^{\circledR} 2000$ reagent (Invitrogen, USA) at 0.5:1.5 $(\mu \mathrm{g}: \mu \mathrm{L})$ in $98 \mu \mathrm{L}$ RPMI-1640 at RT for $20 \mathrm{~min}$, transfected at $37^{\circ} \mathrm{C}$ with $5 \% \mathrm{CO}_{2}$ for $48 \mathrm{~h}$, and screened against $400 \mu \mathrm{g} / \mathrm{mL}$ G418 (Sigma, US). The monoclonal Anxa5- and empty vector-transfected Hca-P cells were obtained using limiting dilution method and named as Hca-P-ANXA5up and Hca-P-mock. ANXA5 expression level was measured by Western blotting assay.

Boyden transwell chamber assay for migration and invasion

24-well transwell units with $8 \mu \mathrm{m}$ I.D. polyester membrane plates (Corning, US) were used for migration assay. $500 \mu \mathrm{L} 20 \%$ FBS (TransGen, China) was loaded into the lower compartment. $2 \times 10^{4}$ cells prepared in $100 \mu \mathrm{L}$ RPMI-1640 from each of Hca-P-mock and Hca-P-ANXA5up were seeded into the upper chamber. Being incubated with $5 \% \mathrm{CO}_{2}$ at $37^{\circ} \mathrm{C}$ for $24 \mathrm{~h}$, the non-migrated cells on the upper surface of filter were wiped off. The migrated cells at the lower surface were fixed in $4 \%$ paraformaldehyde for $20 \mathrm{~min}$, stained in $0.1 \%$ crystal violet for $20 \mathrm{~min}$ and counted with a light microscope at $100 \times$. Triplicate experiments were performed for each assay.

For invasion assay, the inner surface of a 24-well transwell filter unit was first coated with $25 \mu \mathrm{L}$ of ice-cold ECM gel (BD, US, 1:6 dilution with RPMI-1640) at $37^{\circ} \mathrm{C}$ for $5 \mathrm{~h}$, dried at RT and rehydrated in $40 \mu \mathrm{L}$ RPMI-1640 for $30 \mathrm{~min}$. The rest steps were the same in migration assay.

SDS-PAGE and Western blotting (WB) assay

Proteins were extracted from the pellets of different group cells in RIPA buffer containing $1 \mathrm{mM} \mathrm{Na} \mathrm{VO}_{4}, 1 \mu \mathrm{g} / \mathrm{mL}$ leupeptin and $0.5 \mathrm{mM}$ PMSF and centrifuged with 12,000 rpm at $4{ }^{\circ} \mathrm{C}$ for $15 \mathrm{~min}$. Protein concentration was determined by Bradford assay. Being denatured in boiling water for $3 \mathrm{~min}, 30$ $\mu \mathrm{g}$ of protein from each group was separated by $10 \%$ SDS-PAGE. Protein bands were transferred onto nitrocellulose (NC) membrane, blocked in 5\% skim milk in TBST for $2 \mathrm{~h}$ at RT. The NC membranes were then incubated in primary antibodies ANXA5 (1:2000, Proteintech, China), p-MEK (Ser217/ 221) (1:500, Cell Signaling, US), p-ERK1/2 (ERK1:p-Thr202/ Tyr204; ERK2: p-Thr185/Tyr187) (1:500, Cell Signaling, US), ERK1/2 (1:1000, Cell Signaling, US) and E-Cadherin (1:500, Proteintech, China) at $4^{\circ} \mathrm{C}$ for $16 \mathrm{~h}$. Being washed by TBST for $3 \times 10 \mathrm{~min}$, the NC membranes were merged in peroxidase-conjugated goat Anti-Rabbit/Anti-Mouse IgG at RT for $2 \mathrm{~h}$. Then protein bands were visualized by ECL (Advansta, US), detected and quantified using the ChemiDoc ${ }^{\mathrm{TM}} \mathrm{MP}$ system (Bio-Rad, US).

Quantitative real-time PCR ( $q R T-P C R)$ analysis

Total RNA was extracted from each group cells using Trizol $^{\mathrm{TM}}$ reagent (TransGen, China). Reverse transcription of RNA was performed using the EasyScript ${ }^{\circledR}$ gDNA removal and cDNA synthesis kit (TransGen Biotech, China). qRT-PCR was performed using the TransStart ${ }^{\circledR}$ TipTop Green qPCR kit (TransGen Biotech, China). The primers of E47, Slug, Snail, Twsit1, Twist2, ZEB1, and ZEB2 were listed in Table 1. GAPDH was utilized as the internal reference for qRT-PCR. StepOne analysis system was used for quantifying the mRNA level changes of the above molecules between Hca-P-mock and Hca-P-ANXA5up cells.

Table 1. Target gene primers for qRT-PCR.

\begin{tabular}{ll}
\hline Gene & Primer Sequence \\
\hline \multirow{2}{*}{ Snail } & F: 5'-CACCCTCATCTGGGACTCTC-3', \\
& R: 5'-GAGCTTTTGCCACTGTCCTC-3' \\
Slug & F: 5'-CATCCTTGGGGCGTGTAAGTC-3', \\
& R: 5'-GCCCAGAGAACGTAGAATAGGTC-3' \\
E47 & F: 5'-TTCCCTCCCTGACCTCTC-3' \\
& R: 5'-GGCTACTGATGCGATTTCC-3', \\
ZEB1 & F: 5'-ACCGCCGTCATTTATCCT-3' \\
& R: 5'-TTGTCCTCGTTCTTCTCAT-3' \\
ZEB2 & F: 5' -GAGTGAGGTACAAAAGGTTC-3', \\
& R: 5'-CACTACTGGAAGACCGACA-3' \\
Twist1 & F: 5'-GCCCGTGGACAGAGATTC-3' \\
& R: 5'-CTATCAGAATGCAGAGGTGTGG-3', \\
Twist2 & F: 5'-ATTTCCCTCTAAGTGCCT-3' \\
& R: 5'-CTTGGGTTCTTGTTCTGA-3' \\
GAPDH & F: 5'-GGTGAAGGTCGGTGTGAACG-3', \\
& R: 5'-CTCGCTCCTGGAAGATGGTG-3'
\end{tabular}

Data processing and statistical analysis

All the data were presented as mean \pm SD of at least triplicate independent experiments and processed by SPSS 17.0 software for statistical analysis. The difference between 
groups was assessed by paired student's $t$ test. Differences with $p<0.05$ were considered significant.

\section{Results}

ANXA5 overexpression in monoclonal Hca-P-ANXA5up cell line

Empty pCDNA3.1 (+) and recombinant pCDNA3.1 $(+)$-Anxa5 plasmid were constructed into Hca-P cells. The monoclonal Hca-P-mock and Hca-P-ANXA5up cell lines were obtained against G418 screening using limiting dilution method. Empty vector transfection showed no effect on ANXA5 expression in Hca-P. Comparable expression levels of ANXA5 were detected in Hca-P and Hca-P-mock cells (Figure 1). While, compared with Hca-P-mock cells, the protein expression level of ANXA5 in Hca-P-ANXA5up cells increased by $\sim 50.5 \%$ ( $p=0.025$, Figure 1$)$, which ensured the investigation on ANXA5 deregulation on Hca-P malignant behaviours with the underlying action mechanism.

ANXA5 overexpression promotes Hca-P cell migration and invasion

The migration and invasion of tumor cells through the basement membrane is the hallmark of malignancy. The influences of ANXA5 overexpression on the in vitro migration and invasion abilities of Hca-P were investigated by Boyden transwell chamber assays. The migration capacity through the polycarbonate membrane of transwell filter of Hca-P-ANXA5up cells was about 2.5-fold of that of
Hca-P-mock cells ( $p=0.001$, Figure $2 \mathrm{~A}$ ). For invasion assay, compared with Hca-P-mock, a $94.8 \%$ capacity increase for passing through the matrigel-coated transwell filter was measured for Hca-P-ANXA5up cells ( $p=0.003$, Figure 2B). These results suggest ANXA5 promotes the metastasis potential of Hca-P cells.

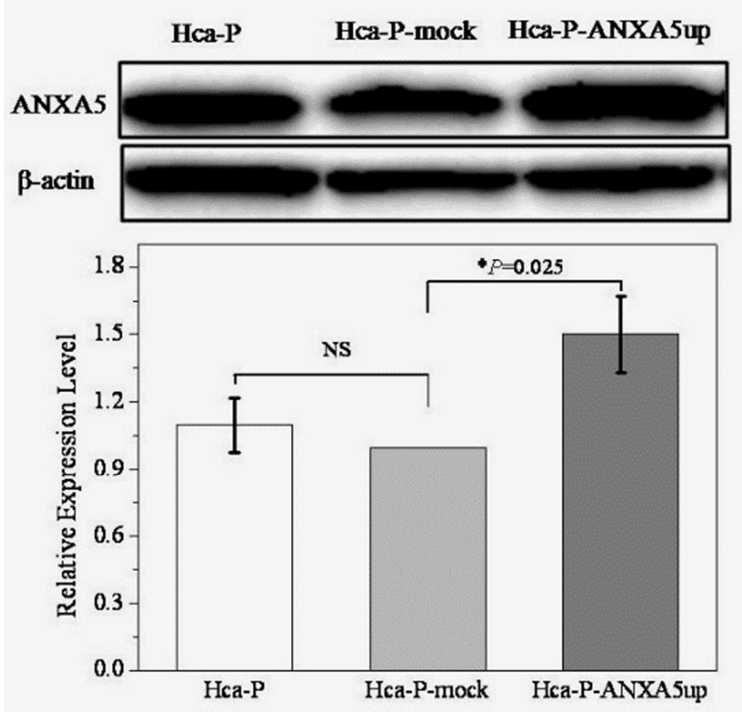

Figure 1. Stable overexpression of ANXA5 in Hca-P. The transfection with empty pCDNA3.1 (+) vector (Hca-P-mock) showed no effect on ANXA5 expression in Hca-P cells. Compared with Hca-P-mock, ANXA5 level increased by $\sim 50.1 \%(p=0.025)$ in the monoclonal Hca-P-ANXA5up cell line.

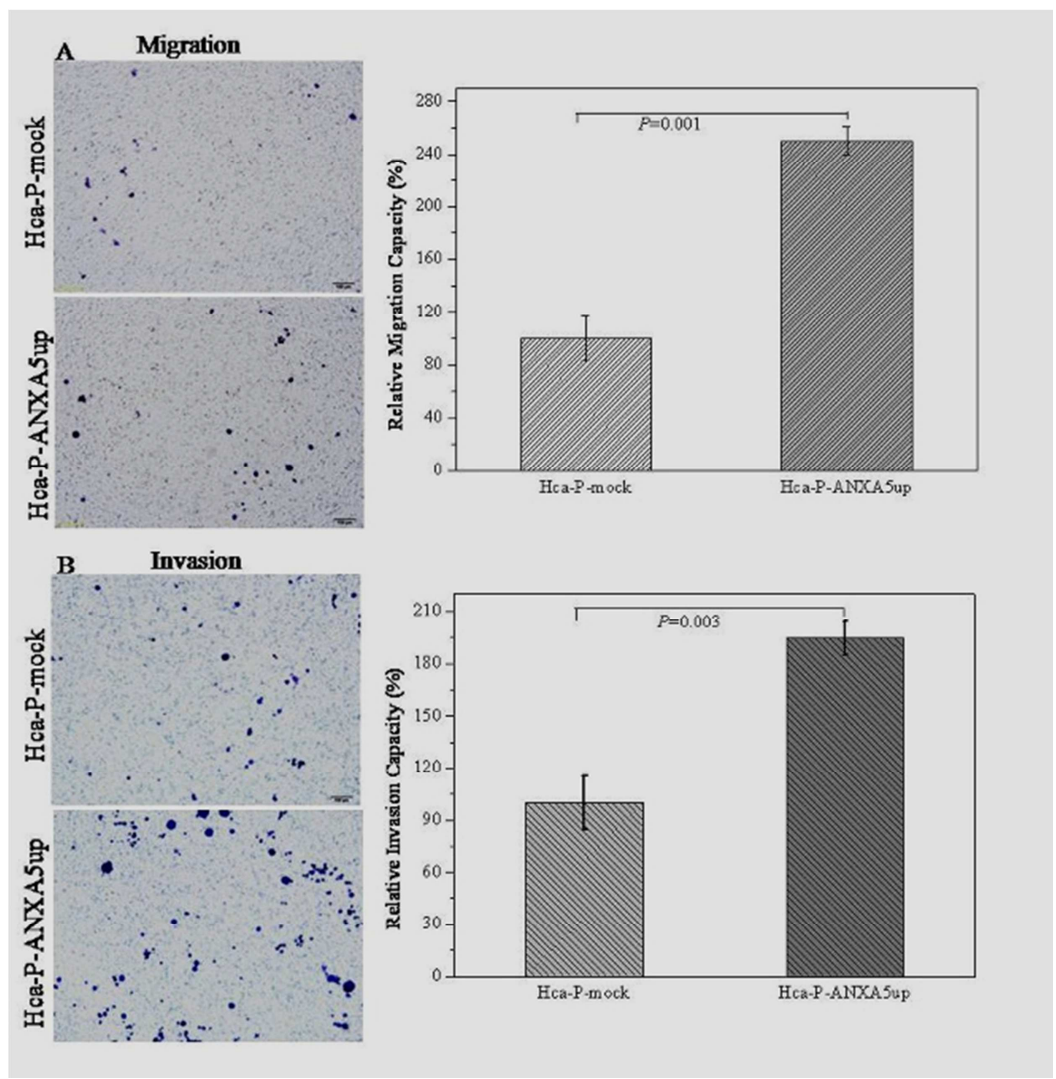

Figure 2. ANXA5 upregulation increased the in vitro migration and invasion capacities of Hca-P. Compared with Hca-P-mock cells, (A) the migration of Hca-P-ANXA5up cells increased by $\sim 150.6 \%(p=0.001)$ and $(B)$ the invasion of Hca-P-ANXA5up increased by $\sim 94.8 \%(p=0.003)$. 
Anxa5 overexpression activates ERK signaling pathway
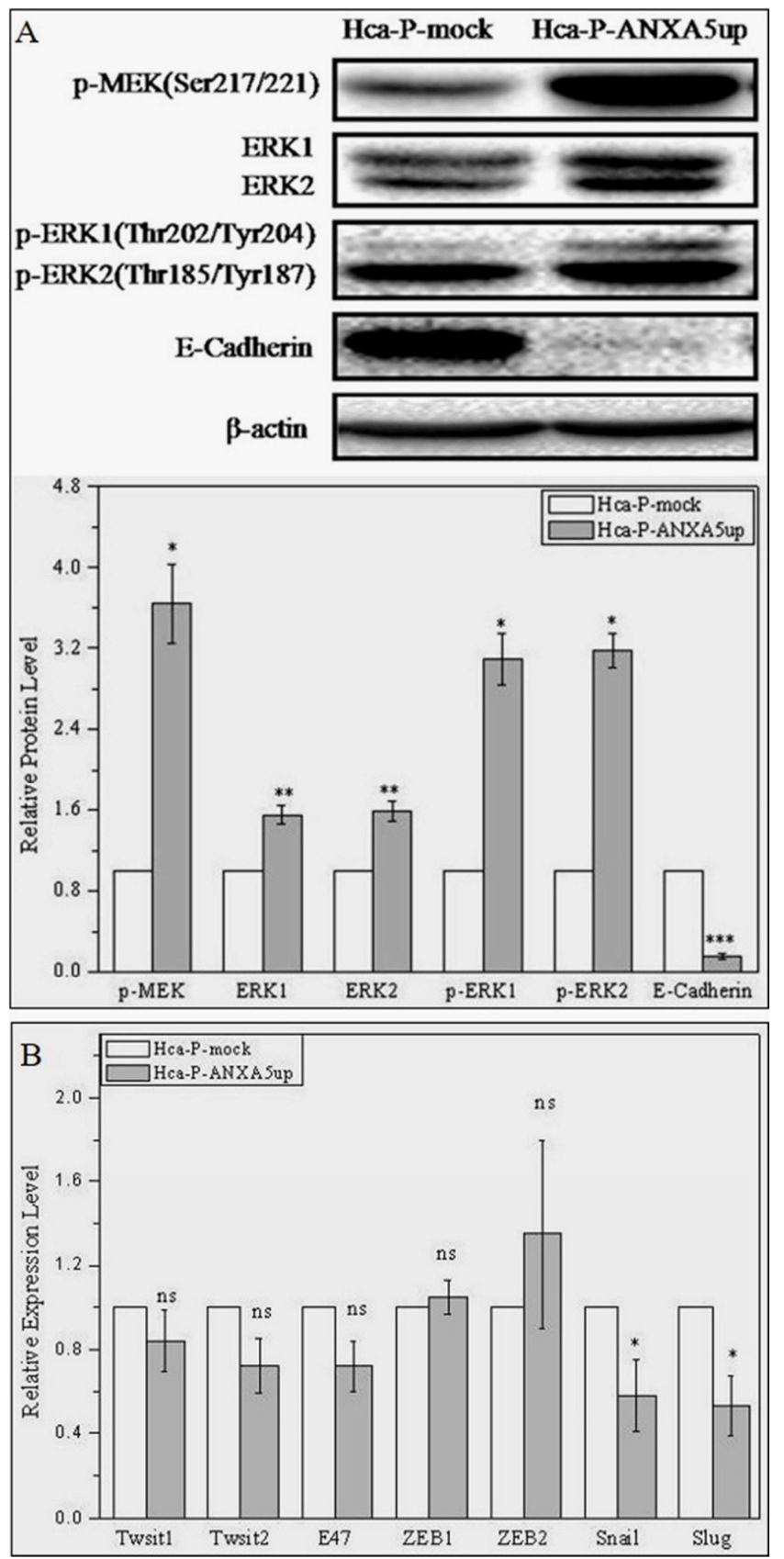

Figure 3. The influence of ANXA5 overexpression on key molecules in ERK pathway and transcription suppressors. ANXA5 overexpression in Hca-P (A) increased protein levels of p-MEK (Ser217/221), ERK1, ERK2, p-ERK1 (Thr202/Tyr204) and p-ERK2 (Thr185/Tyr187), and decreased protein expression of E-Cadherin. ${ }^{* * *}$ and ${ }^{* * *}$ refer to $p$ values $<0.05,0.01$ and 0.001 ; (B) decreased Snail and Slug expressions and showed no effects on Twist 1 , Twist 2, E47, ZEB1 and ZEB2. ns refers to non-significance.

WB assay was performed to measure the expression levels of key molecules in ERK transduction pathway including p-MEK (Ser217/221), ERK1, ERK2, p-ERK1 (Thr202/Tyr204) and p-ERK2 (Thr185/Tyr187) in Hca-P-mock and Hca-P-ANXA5up cells. Compared with Hca-P-mock cells, the levels of p-MEK (Ser217/221), ERK1, ERK2, p-ERK1 (Thr202/ Tyr204) and p-ERK2
(Thr185/Tyr187) increased by $264 \%(p<0.05), 55 \%(p<0.01)$, $59 \%(p<0.01), 209 \%(p<0.05)$ and $218 \%(p<0.05)$ (Figure $3 \mathrm{~A})$. The isoforms of ERK1 (44 kDa) and ERK2 (42 kDa) as well as their phosphorylated isoforms p-ERK1 (Thr202/Tyr204) and p-ERK2 (Thr185/Tyr187) were cogradiently activated following ANXA5 upregulation. ANXA5 enhances the malignant behaviours of Hca-P via activating $\mathrm{p}$-MEK-ERK/p-ERK pathway.

ANXA5 overexpression suppresses E-cadherin expression

E-cadherin is important in tumorigenesis. Its suppression or loss is commonly involved in tumor development, invasion and metastasis. The overexpression of ANXA5 in Hca-P cells resulted in decreased expression of E-cadherin. Compared with Hca-P-mock cells, E-cadherin level in Hca-P-ANXA5 up cells was reduced by about $84 \%(p<0.001$, Figure $3 \mathrm{~A})$. E-cadherin suppression by ANXA5 upregulation contributes to enhanced malignancy of Hca-P cells.

ANXA5 overexpression reduces Snail and Slug levels

The influence of ANXA5 overexpression on the transcription suppressors including E47, Snail, Slug, Twist 1, Twist 2, ZEB1 and ZEB2 was investigated. The qRT-PCR assays indicated, except for Snail and Slug, no apparent expression level changes of E47, Twist 1, Twist 2, ZEB1 and ZEB2 were detected in the Hca-P-ANXA5up cells compared with the Hca-P-mock cells (Figure 3B). The upregulation of ANXA5 in Hca-P cells led to the decreases of Snail and Slug levels by about $42 \%$ and $47 \%(p<0.05$, Figure $3 B)$. ANXA5 overexpression probably suppresses Snail and Slug expressions, consequently, inhibits E-cadherin for decreasing migration and invasion malignant behaviour of Hca-P cells.

\section{Discussion}

Annexins are a superfamily of $\mathrm{Ca}^{2+}$-regulated phospholipid -binding proteins. There are 12 members in annexin A family, A1-A11 and A13, in vertebrate and mammalian. The dysexpressions and/or subcellular redistributions of annexins are involved in tumor cell proliferation, tumor invasion, metastasis, angiogenesis and drug resistance [7, 10, 18-20]. As a member of annexin A family that was discovered as a vascular anticoagulant in 1985 [21], annexin A5 (ANXA5) is involved in tumor progression, invasion, metastasis, drug resistance and treatment [7-9, 14, 17]. Using Hca-P and Hca-F, a pair of syngenetic murine hepatocarcinoma cell lines with different LNM potentials, we demonstrated ANXA5 acting as a promoter in tumorigenesis and lymphatic metastasis of hepatocarcinoma $[10,13,14,22]$. We also showed ANXA5 upregulation enhanced the in vivo tumorigenicity, LNM rate and level of Hca-P-transplanted mice [7, 14]. Current work investigated the underlying promoting mechanism of ANXA5 on the malignancy of Hca-P cells. Owing low LNM rate without dissemination to other organs, Hca-P has been proved by our group as an ideal cell model both for tumor malignancy, initial (low) and specific lymphatic metastasis of hepatocarcinoma $[10-13,14,20,23-24]$. As schemed in Figure 4, current work showed ANXA5 overexpression in 
Hca-P led to enhanced activation of p-MEK-ERK signaling, triggered downregulations of Snail and Slug, decreased the expression of E-cadherin and resulted in increased malignant behaviours of Hca-P cells.

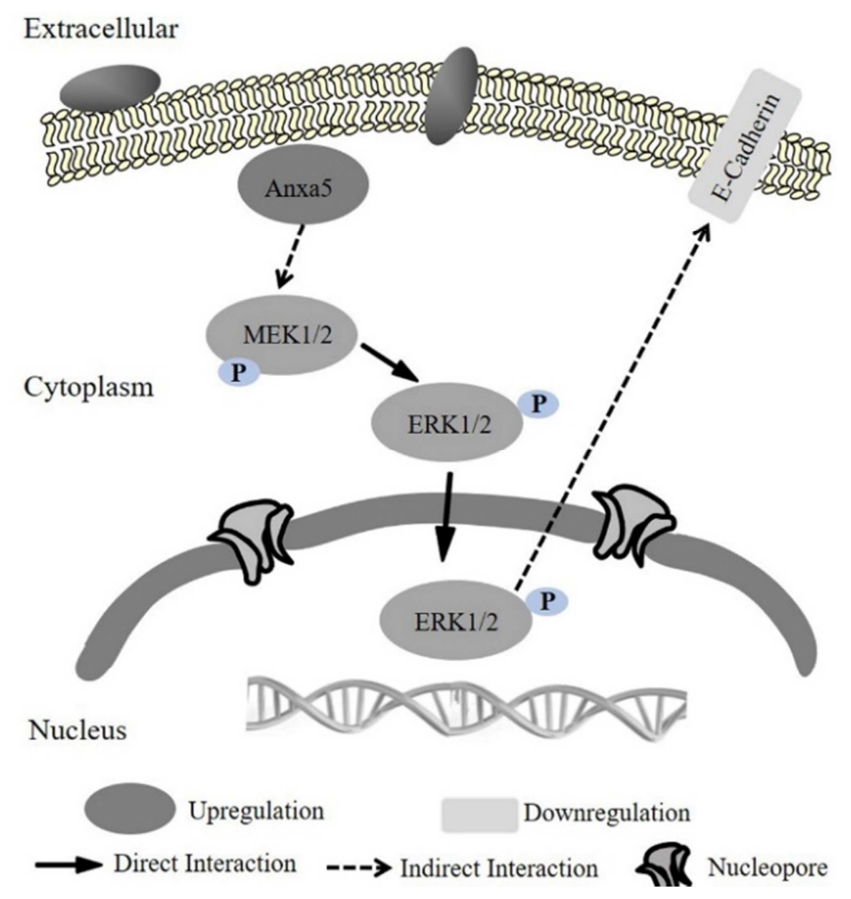

Figure 4. Schematic regulation mechanism of ANXA5 on the migration and invasion of Hca-P cells. ANXA5 overexpression activated the phosphorylation of MEK1/2 (Ser217/221), subsequently, enhanced both the expression levels of ERK1 and ERK2 and phosphorylation levels of p-ERK1 (Thr202/Tyr204) and p-ERK2 (Thr185/ Tyr187), which potentially triggered downregulations of E-cadherin, Snail and Slug by promoting malignant potentials of hepatocarcinoma cells.

We established a monoclonal Hca-P-ANXA5up cell line with a stable ANXA5 upregulation of about 50.5\% (Figure 1), which made feasible for investigating the deregulation molecular mechanism of ANXA5 in Hca-P cells. Compared with Hca-P-mock, the in vitro migration and invasion capacities of Hca-P-ANXA5up cells elevated by $150.6 \%$ $(p=0.001$, Figure 2A) and $94.8 \%(p=0.003$, Figure $2 \mathrm{~B})$. ERK/MAPK pathway is involved in the proliferation, metastasis and drug resistance of various cancers [25-27]. The phosphorylation of MEK at the site Ser217/221 leads to the phosphorylation of ERK1/2, a key molecule in ERK pathway, at Thr202/Tyr204. Then, p-ERK1/2 (Thr202/Tyr204) dissociated from p-MEK (Ser217/221) and translocated into nucleus for functionality $[27,28]$. ERK1/2 was overexpressed in HCC cell line [16]. ANXA5 upregulation in MCF-7 cells suppressed Ras/Raf/MEK/ERK pathway activated by 12-O-tetradecanoylphorbol-13-acetate (TPA) [17]. Work from our group also showed ANXA5 knockdown decreased the in vitro malignant behaviours of Hca-F through ERK2/p-ERK2/c-Jun pathway [22]. Herein, ANXA5 overexpression in Hca-P activated p-MEK-ERK/p-ERK pathway. The expression levels of p-MEK (Ser217/221), ERK1, ERK2, p-ERK1 (Thr202/Tyr204) and p-ERK2 (Thr185/Tyr187) in Hca-P-ANXA5up cells were 3.64-, 1.55-,
1.59-, 3.09- and 3.18-fold of those in Hca-P-mock cells (Figure 3A). Clearly, ANXA5 overexpression enhanced the activation of MEK-ERK signaling transduction contributing to the higher malignancy of Hca-P cells (Figure 4).

Cancer metastasis mainly contributes from the invasion and migration abilities of tumor cells. Epithelial-mesenchymal transition (EMT) is crucial for the dissemination and invasion of certain cancers [29]. E-cadherin suppression or loss is an important induction factor of EMT in tumorigenesis [15, 28, 30-33]. The requisition of E-cadherin suppressed the ability of metastasis tumor cells [33]. ERK signaling pathway mediated E-cadherin in hepatoma cells [16, 30]. We proposed that ANXA5-overexpression-enhanced ERK activity might induce E-cadherin deregulation. The result showed the level of E-cadherin in Hca-P-ANXA5up was only about $16 \%$ of that in Hca-P-mock cells $(p<0.001$, Figure 3A). Probably, E-cadherin suppression induced by ANXA5 overexpression contributes to increased malignant potential of Hca-P cells (Figure 4) under the mediation of $\mathrm{p}-\mathrm{MEK} / \mathrm{ERK}$ signaling transduction.

More commonly reported as a suppresser for tumor invasion and metastasis, the activity of E-cadherin was more commonly reported to be negatively regulated by transcription repressors E47, Snai1, Slug, Twist 1, Twist2, ZEB1 and ZEB2 [34-37]. ANXA5 overexpression did not lead to expression increases of above molecules in Hca-P-ANXA5up cells by qRT-PCR assay (Figure 3B). While interestingly, the expression levels of Snail and Slug were decreased by $42 \%$ and $47 \%$ in Hca-P-ANXA5up cells than Hca-P-mock cells $(p<0.05$, Figure 3B). In non-small cell lung cancer cell lines, E-cadherin, Snail and Slug were simultaneously decreased with Musashi-2 depletion [38]. E-cadherin and Snail expressions were both enhanced in hepatic cells of hepatitis-hepatom mouse induced by inflammation [39]. This work showed that Snail and Slug downregulations are triggered in enhanced malignant potentials of Hca-P cells induced by ANXA5 overexpression, which was consistent with previous study that ANXA5 knockdown in Hca-F cells resulted in simultaneous upregulations of E-cadherin, Snail and Slug [22]. The role and mediation mechanism of ANXA5 deregulation in tumor invasion and metastasis are worthy of further investigation.

\section{Conclusion}

ANXA5 is linked to hepatocarcinoma malignancy. The stable overexpression of ANXA5 promotes the in vitro migration and invasion abilities of Hca-P cells. The expression levels of p-MEK (Ser217/221), ERK1, ERK2, p-ERK1 (Thr202/Tyr204) and p-ERK2 (Thr185/Tyr187) were significantly elevated in cancer cells following ANXA5 overexpression. The enhanced activation of p-MEK-ERK signaling triggered the down-regulations of E-Cadherin, Snail and Slug. ANXA5 deregulation induced the malfunctions of these molecules in mediating the malignant behaviours Hca-P cells. ANXA5 is a potential target in tumor malignancy and lymphatic metastasis of hepatocarcinoma. 


\section{Acknowledgements}

This work was supported by grants from National Natural Science Foundation of China (81672737, 81171957 and 81272186). The authors declare no conflict of interest in current work.

\section{References}

[1] W. Chen, R. Zheng, P. D. Baade, S. Zhang, H. Zeng, F. Bray, A. Jemal, X. Yu, and J. He, "Cancer statistics in China, 2015," CA Cancer J. Clin. New York, 2016, vol. 66, pp. 115-132.

[2] J. Zhang and Y. Qian, "Treatment options of postoperative recurrence of hepatocellular carcinoma," Chin. J. Clinicians. Beijing, 2015, vol. 9, pp. 3319-3321.

[3] Z. Tang, S. Ye, Y. Liu, L. Qin, H. Sun, Q. Ye, L. Wang, J. Zhou, S. Qiu, Y. Li, X. Ji, H. Liu, J. Xia, Z. Wu, J. Fan, Z. Ma, X. Zhou, Z. Lin, and K. Liu, "A decade's studies on metastasis of hepatocellular carcinoma," J. Cancer Res. Clin. Oncol. Berlin, 2004, vol. 130, pp. 187-196.

[4] S. D. Nathanson, "Insights into the mechanisms of lymph node metastasis," Cancer. New York, 2003, vol. 98, pp. 413-423.

[5] S. Li and Q. Li, "Cancer stem cells, lymphangiogenesis, and lymphatic metastasis," Cancer Letter. Amsterdam, 2014, vol. 357, pp. 438-447.

[6] S. Stacker, M. Baldwin, and M. Achen, "The role of tumor lymphangiogenesis in metastatic spread," FASEB J. Bethesda, 2002, vol. 16, pp. 922-934.

[7] B. Peng, C. Guo, H. Guan, S. Liu, and M-Z. Sun, "Annexin A5 as a potential marker in tumors," Clin. Chim. Acta. Amsterdam, 2014, vol. 427, pp. 42-48.

[8] G. Xue, L. Hao, F. Ding, Q. Mei, J. Huang, C. Fu, H. Yan, and S. Sun, "Expression of annexin A5 is associated with higher tumor stage and poor prognosis in colorectal adenocarcinomas," J. Clin. Gastroenterol. New York, 2009, vol. 43, pp. 831-837.

[9] U. Rajcevic, K. Petersen, J. Knol, M. Loos, S. Bougnaud, O. Klychnikov, K. Li, T. Pham, J. Wang, H. Miletic, Z. Peng, R. Bjerkvig, C. Jimenez, and S. Niclou, "iTRAQ-based proteomics profiling feveals increased metabolic activity and cellular cross-talk in angiogenic compared with invasive glioblastoma phenotype," Mol. Cell Proteomics. Bethesda, 2009, vol. 8, pp. 2595-2612.

[10] M-Z. Sun, S. Liu, J. Tang, Z. Wang, X. Gong, C. Sun, and F. Greenaway, "Proteomics analysis of two mice hepatocarcinoma ascites syngeneic cell lines with high and low lymph node metastasis rates provide potential protein markers for tumor malignancy attributes to lymphatic metastasis," Proteomics. Weinheim, 2009, vol. 9, pp. 3285-3302.

[11] S. Liu, C. Guo, J. Wang, B. Wang, H. Qi, and M-Z. Sun, "ANXA11 regulates the tumorigenesis, lymph node metastasis and 5-fluorouracil sensitivity of murine hepatocarcinoma Hca-P cells by targeting c-Jun," Oncotarget. Albany, 2016, vol. 7, pp. 16297-16310.

[12] J. Shi, L. Meng, M-Z. Sun, C. Guo, X. Sun, Q. Lin, and S. Liu, "CRKL knockdown promotes in vitro proliferation, migration and invasion, in vivo tumor malignancy and lymph node metastasis of murine hepatocarcinoma Hca-P cells," Biomed.
Pharmacother. New York, 2015, vol. 71, pp. 84-90.

[13] S. Liu, M-Z. Sun, J. Tang, Z. Wang, C. Sun, and F. T. Greenaway, "High-performance liquid chromatography/nano-electrospray ionization tandem mass spectrometry, two-dimensional difference in-gel electrophoresis and gene microarray identification of lymphatic metastasis-associated biomarkers," Rapid Commun. Mass Spectrom. Chichester, 2008, vol. 22, pp. 3172-3178.

[14] B. Peng, S. Liu, C. Guo, X. Sun, and M-Z. Sun, "Anxa5 level is linked to in vitro and in vivo tumor malignancy and lymphatic metastasis of murine hepatocarcinoma cell," Future Oncol. London, 2016, vol. 12, pp. 31-42.

[15] G. L. Johnson and R. Lapadat. "Mitogen-activated protein kinase pathways mediated by ERK, JNK, and p38 protein kinases," Science. New York, 2002, vol. 298, pp. 1911-1912.

[16] A. Mehdizadeh, M. H. Somi, M. Darabi, and M. Jabbarpour-Bonyadi, "Extracellular signal -regulated kinase 1 and 2 in cancer therapy: a focus on hepatocellular carcinoma," Mol. Biol. Rep. Dordrecht, 2016, vol. 43, pp. 107-116.

[17] H. Sato, H. Ogata, and L. M. De Luca, “Annexin V inhibits 12-O-tetradecanoylphorbol-13-acetate-induced activation of Ras/ extracellular signal-regulated kinase (ERK) signaling pathway upstream of Shc in MCF-7 cells," Oncogene. Basingstoke, 2000, vol. 19, pp. 2904-2912.

[18] M. A. Lizarbe, J. I. Barrasa, N. Olmo, F. Gavilanes, and J. Turnay, "Annexins-phospholipid interactions. Functional implications," Int. J. Mol. Sci. Basel, 2013, vol. 14, pp. 2652-2683.

[19] A. Laohavist and J. M. Davies, "Annexins," New Phytol. London, 2011, vol. 189, pp. 40-53.

[20] M-Z. Sun, S. Liu, and J. Tang. "Proteomics investigation of mouse hepatocarcinoma cell lines with different lymph node metastasis capacities," Chem. J. Chin. Univ. Beijing, 2009, vol. 30, pp. 517-524.

[21] H. Nakao, M. Watanabe, and M. Maki, "A new function of calphobind I (annexin V),” Eur. J. Biochem. Berlin, 1994, vol. 223, pp. 901-908.

[22] X. Sun, B. Wei, S. Liu, C. Guo, N. Wu, Q. Liu, and M-Z. Sun, "Anxa5 mediates in vitro malignant behaviours of murine hepatocarcinoma Hca-F cells with high lymph node metastasis potential preferentially via ERK2/p-ERK2/c-Jun/p-c-Jun (Ser73) and E-cadherin," Biomed Pharmacother. New York, 2016, vol. 84, pp. 645-654.

[23] Q. Lin, M-Z. Sun, C. Guo, J. Shi, and X. Chen, "CRKL overexpression suppresses in vitro proliferation, invasion and migration of murine hepatocarcinoma Hca-P cells," Biomed Pharmacother. New York, 2015, vol. 69, pp. 11-17.

[24] J. Wu, J. Meng, Y. Du, Y. Huang, Y. Jin, J. Zhang, B. Wang, Y. Zhang, M-Z. Sun, and J. Tang, "RACK1 promotes the proliferation, migration and invasion capacity of mouse hepatocellular carcinoma cell line in vitro probably by PI3K/ Rac1 signaling pathway," Biomed. Pharmacother. New York, 2013, vol. 67, pp. 313-319.

[25] G. Guo, W. Yao, Q. Zhang, and Y. Bo, "Oleanolic acid suppresses migration and invasion of malignant glioma cells by inactivating MAPK/ERK signaling pathway," PLoS One. San Francisco, 2013, vol. 8, pp. e72079. 
[26] P. Wang, Y. Zeng, T. Liu, C. Zhang, P. Yu, Y. Hao, H. Luo, and G. Liu, "Chloride intracellular channel 1 regulates colon cancer cell migration and invasion through ROS/ERK pathway," World J. Gastroenterol. Beijing, 2014, vol. 20, pp. 2071-2078.

[27] Y. Sun, W. Liu, T. Liu, X. Feng, N. Yang, and H. Zhou, "Signaling pathway of MAPK/ERK in cell proliferation, differentiation, migration, senescence and apoptosis," J. Recept Signal Transduct Res. New York, 2015, vol. 35, pp. 600-604.

[28] Y. Mebratu, and Y. Tesfaigzi, "How ERK1/2 activation controls cell proliferation and cell death is subcellular localization the answer?" Cell Cycle. Georgetown, 2009, vol. 8, pp. 11681175 .

[29] M. Yilmaz, G. Christofori, and F. Lehembre, "Distinct mechanisms of tumor invasion and metastasis," Trend Mol. Med. Oxford, 2007, vol. 13, pp. 535-541.

[30] H. Semb and G. Christofori, "The tumor-suppressor function of E-cadherin,” Am. J. Hum. Genet. New York, 1998, vol. 63, pp. 1588-1593.

[31] O. Schmalhofer, S. Brabletz, and T. Brabletz, "E-cadherin, $\beta$-catenin, and ZEB1 in malignant progression of cancer," Cancer Met. Rev. The Hague, 2009, vol. 28 pp. 151-166.

[32] M. R. Schneider and F. T. Kolligs, "E-cadherin's role in development, tissue homeostasis and disease: Insights from mouse models tissue-specific inactivation of the adhesion protein E-cadherin in mice reveals its functions in health and disease," Bioessays. Cambridge, 2015, vol. 37, pp. 294-304.

[33] T. T. Onder, P. B. Gupta, and S. A. Mani, "Loss of E-Cadherin promotes metastasis via multiple downstream transcriptional pathways," Cancer Res. Chicago, vol. 68, pp. 3645-3654.
[34] E. Howard, K. Cam, Y. Wong, J. Yang, E. S. Lander, and R. A. Weinberg, "E-cadherin upregulation as a therapeutic goal in cancer treatment," Mini. Rev. Med. Chem. Hilversum, 2008, vol. 8, pp. 496-518.

[35] T. Chou, W. Chen, A. Lee, S. Hung, N. Shih, and M. Chen, "Clusterin silencing in human lung adenocarcinoma cells induces a mesenchymal-to-epithelial transition through modulating the ERK/Slug pathway," Cell Signal. Oxford, 2009, vol. 21, pp. 704-711.

[36] H. Peinado, F. Portillo, and A. Cano, "Transcriptional regulation of cadherins during development and carcinogenesis,” Int. J. Dev. Biol. Vizcaya, 2004, vol. 48, pp. 365-375.

[37] T. Bhatt, A. Rizvi, S. Batta, S. Kataria, and C. Jamora, "Signaling and mechanical roles of E-cadherin," Cell Commun. Adhes. Basingstoke, 2013, vol. 20, pp. 189-199.

[38] A. Kudinov, A. Deneka, A. S. Nikonova, T. N. Beck, Y. Ahn, and X. Liu, "Musashi-2 (MSI2) supports TGF- $\beta$ signaling and inhibits claudins to promote non-small cell lung cancer (NSCLC) metastasis," Proc. Natl Acad. Sci. USA. Washington, 2016, vol. 113, pp. $6955-6960$.

[39] K. Lu, G. Liu, L. Yang, F. Liu, L. Gao, J. Shi, X. Deng, Q. Li, D. $\mathrm{Xu}$, and S. Shi, "Sustainable inflammation transforms hepatic cells by causing oxidative stress injury and potential epithelial-mesenchymal transition," Int. J. Oncol. Athens, 2016, vol. 49 , pp. 971-980. 